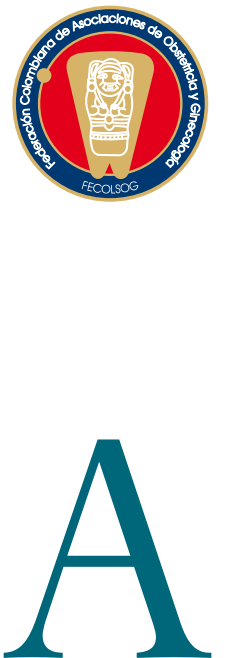

\title{
EDITORIAL
}

\section{COMENTARIOS EDITORIALES}

\section{DUPLICACIÓN, REDUNDANCIA Y ÉTICA EN LAS PUBLICACIONES MÉDICAS*}

raíz de la reciente publicación del trabajo de Rodríguez J, Prieto S, Ortiz L, Bautista A, Bernal P, Vargas

N. Diagnóstico matemático de la monitoría fetal. Aplicando la Ley de Zipf-Mandelbrot; en el número 2 del volumen 54 de nuestra revista ${ }^{1}$ y la simultánea aparición en la Revista Colombiana de Obstetricia y Ginecología de: Rodríguez J, Prieto S, Ortiz L, Bautista A, Álvarez L, Correa C, Avilán N. Diagnóstico matemático de la monitoría fetal con la Ley de Zipf-Mandelbrot y la teoría de los sistemas dinámicos aplicados a la fisiología cardíaca; ${ }^{2}$ el editor desea precisar como es costumbre en las revistas médicas que: ${ }^{3}$

1.- Todo trabajo científico recibido para evaluación del comité editorial, se acompaña de una carta firmada por todos los autores donde se establece que el manuscrito no ha sido presentado a otra revista.

2.- El proceso de revisión por pares es anónimo y que durante él se mantiene comunicación con los autores a fin de corregir, adicionar o ampliar los conceptos contenidos en el trabajo analizado.

3.- Una vez aceptado para publicación, los autores reciben notificación de la fecha probable de publicación (volumen y año).

La amplia homología de las publicaciones mencionadas y en especial de la metodología utilizada, los grupos de pacientes y las conclusiones, permiten aseverar que se trata de una publicación redundante.

Este hecho, poco deseable, en el ejercicio como editor de revistas médicas permite revisar algunos aspectos recientes sobre publicación redundante y duplicada. Se entiende por la primera, la aparición de material que ya tiene derechos (copyrighted material) al cual se agrega información nueva o no publicada. La publicación duplicada es aquel artículo idéntico o superpuesto con otro ya publicado, se aclare o no esta circunstancia. En ambas ocasiones se consumen los esfuerzos de los editores, de los revisores y de las publicaciones. ${ }^{4}$

En el primer caso se induce, además de los problemas de derechos, a error por cuanto la redundancia divide el cuerpo de información en lugar de consolidarla y puede inducir a confusión en los metaanálisis y demás estudios que utilizan información publicada como fuente de trabajo. Como lo demostró el estudio de Von Elm y otros; hay hasta 8 por ciento de artículos redundantes y hasta 9 por ciento de los pacientes reevaluados. En el segundo se intenta una abultada producción "científica" que a veces se justifica por la necesidad de publicar para mantener o mejorar una posición académica (publicar o perecer), para solicitar becas y financiaciones, etc. ${ }^{5,6}$

Es importante establecer la necesidad de una ética en el sometimiento de originales a los comités editoriales de las revista y de evitar a ultranza todas las acciones que puedan comprometer la ética de las publicaciones. La mala práctica científica incluye desde la publicación redundante y duplicada en el extremo inferior, hasta el fraude y el plagio en el superior. Existen claras indicaciones para evitar estas circunstancias que autores y editores deben mantener presentes. ${ }^{7,8}$

Autorizado para reproducción. Publicado originalmente en la Rev Fac Med Univ Nac Colomb 2006;54:238-9. 
Es importante respetar los derechos de los autores y disminuir en cuanto sea posible el tiempo para que un trabajo aparezca publicado como lo proponen Torgerson y otros, pero no es menos importante respetar a los lectores y defender la tradicional "buena fe manifiesta”, aún más necesaria en un mundo científico y globalizado. Invitamos a nuestros autores y lectores a revisar las indicaciones éticas para publicaciones disponibles en COPE.

Germán Enrique Pérez Romero Profesor Medicina Universidad Nacional de Colombia Editor Correspondencia: geperezr@unal.edu.co

\section{REFERENCIAS}

1. Rodríguez J, Prieto S, Ortiz L, Bautista A, Bernal P, Vargas N. Diagnóstico matemático de la monitoría fetal. Aplicando la Ley de Zipf-Mandelbrot. Rev Fac Med Univ Nac Colomb 2006;54:96-107.

2. Rodríguez J, Prieto S, Ortiz L, Bautista A, Álvarez L, Correa C, Avilán N. Diagnóstico matemático de la monitoría fetal con la Ley de Zipf-Mandelbrot y la teoría de los sistemas dinámicos aplicados a la fisiología cardíaca. Rev Colomb Obstet Ginecol 2006;57:88-99.

3. Folle LE, Montalbán A. Presentación de artículos originales; evitemos la duplicación o repetición. Rev Med Uruguay 1996,12-3-5.

4. Benos DJ, Fabres J, Farmer J, Gutierrez JP, Hennessy K, Kosek D, Lee JH, Olteanu D, Russell T, Shaikh F, Wang K. Ethics and scientific publication. Adv Physiol Educ 2005; 29:59-74.

5. Gollogly L, Momen H. Ethical dilemmas in scientific publication: pitfalls and solutions for editors. Rev Saude Publica 2006;40:24-29.

6. Von Elm E, Poglia G, Walter B, Tramer MR. Different patterns of duplicate publication: an analisis of articles used in systematic reviews. JAMA 2004;291:974-80.

7. Bannura G. Duplicación en la presentación y publicación de un artículo médico. Rev Chilena de Cirugía 2006;58:75-76.

8. Balch CM. Duplicate submission and scientific fraud: the Annals of Surgical Oncology perspective. Ann Surg Oncol 2001;8:38

9. Committee on Publication Ethics - COPE. Guidelines on good publication and the Code of Conduct. Disponible en http://www.publicationethics.org.uk/ guidelines. Visitado Nov 27 de 2006. 